\title{
Profiling Viral Infections in Grapevine Using a Randomly Primed Reverse Transcription-Polymerase Chain Reaction/ Macroarray Multiplex Platform
}

\author{
J. R. Thompson, Marc Fuchs, Heather McLane, Fevziye Celebi-Toprak, \\ Kael F. Fischer, Jamie L. Potter, and Keith L. Perry
}

First, third, and seventh authors: Department of Plant Pathology and Plant-Microbe Biology, Cornell University, 334 Plant Science, Ithaca, NY 14850; second author: Department of Plant Pathology and Plant-Microbe Biology, Cornell University, New York State Agricultural Experiment Station, Geneva, NY 14456; fourth author: Department of Biology, Pamukkale University, Denizli 20070, Turkey; fifth author: Department of Pathology, University of Utah, 15 North Medical Drive East, Salt Lake City 84112; and sixth author: Houghton College, One Willard Avenue, Houghton, NY 14744.

Accepted for publication 20 August 2013.

\begin{abstract}
Thompson, J. R., Fuchs, M., McLane, H., Celebi-Toprak, F., Fisher, K. F., Potter, J. L., and Perry, K. L. 2014. Profiling viral infections in grapevine using a randomly primed reverse transcription-polymerase chain reaction/macroarray multiplex platform. Phytopathology 104:211-219.

Crop-specific diagnostics to simultaneously detect a large number of pathogens provides an invaluable platform for the screening of vegetative material prior to its propagation. Here we report the use of what is to-date the largest published example of a crop-specific macroarray for the detection of 38 of the most prevalent or emergent viruses to infect grapevine. The reusable array consists of 1,578 virus-specific 60 to $70 \mathrm{mer}$ oligonucleotide probes and 19 plant and internal control probes spotted onto an

$18 \times 7 \mathrm{~cm}$ nylon membrane. In a survey of 99 grapevines from the United States and Europe, virus infections were detected in 46 selections of Vitis vinifera, $V$. labrusca, and interspecific hybrids. The majority of infected vines (30) was singly infected, while 16 were mixed-infected with viruses from two or more families. Representatives of the four main virus families Betaflexiviridae, Closteroviridae, Secoviridae, and Tymoviridae present in grapevines were found alone and in combination, with a notable bias in representation by members of the family Tymoviridae. This work demonstrates the utility of the macroarray platform for the multiplex detection of viruses in a single crop, its potential for characterizing grapevine virus associations, and usefulness for rapid diagnostics of introduced material in quarantine centers or in certification programs.
\end{abstract}

There is more land in the world devoted to grapevine cultivation than to any other fruit crop with the exception of citrus. As an agricultural commodity, grape ranks 14th in the world with a production of 58 million tons valued at just under $\$ 40$ billion annually (http://faostat.fao.org). Knowledge of the viruses that infect grapevine and their effects on plant health has gained momentum in recent years. This has been in part because of a number of factors: (i) an upward trend in global production coupled with the continuous exchange of propagative material at both international and national levels has increased the potential for virus spread, (ii) marked advances in detection technologies are gradually leading to improvements in diagnostics and monitoring of disease spread, and (iii) large-scale sequencing technologies are revealing previously unrecognized viruses. At least 58 virus species are known to be associated with grapevine (40), their infection having a range of effects that reduce both quantity - from a yield loss (up to 68\% has been reported [40]) and quality, causing a reduction of Brix values and an increased acidity of fruit juice (30). New viruses are being discovered at a consistent rate. Until 2011 all grape viruses described had been RNA encoded; since then two novel DNA viruses have been characterized, Grapevine vein-clearing virus (GVCV) (59) and Grapevine red blotch-associated virus (GRBaV, originally desig-

Corresponding author: J. R. Thompson; E-mail address: jrt36@ cornell.edu

* The $e$-Xtra logo stands for "electronic extra" and indicates that the online version contains two supplemental figures and one supplemental table.

http://dx.doi.org/10.1094/PHYTO-06-13-0166-R

(c) 2014 The American Phytopathological Society nated Grapevine cabernet franc-associated virus) (6,32). Most grapevine viruses fall into one of four families: Betaflexiviridae, Closteroviridae, Secoviridae, and Tymoviridae. Additionally, there are less common viruses outside of these families that have been detected in grape, such as Alfalfa mosaic virus and Tomato spotted wilt virus, although their impact on and distribution in the crop has not been clearly established. Primary spread of viruses is due to the planting of new vineyards with material derived from noncertified or poorly certified planting stock, while secondary spread, via vectors $(18,22,33,54,55)$, can occur any time thereafter, resulting in a progressive spread of disease during the lifetime of the vineyard $(9,13,56)$. The principal vectors involved in the transmission of the most common and economically important grape viruses, mealybugs and nematodes, have limited motility, but are difficult to control. (15). Therefore, prevention based on the use of planting material derived from certified and disease-tested stocks is the control method of choice.

The development of robust and reliable detection methods for routine diagnostics in grapevine has revolved mainly around enzyme-linked immunosorbent assay (ELISA) and reverse transcription (RT)-polymerase chain reaction (PCR). Sensitivity can be a limitation for ELISA, and both methods are subject to potential false negatives due to variations in the target molecule, be it failure of the antibody to recognize an altered epitope in the former or primer mismatching leading to low or no amplification in the latter. Next generation sequencing promises to provide broad screens for plant viruses, but presently its implementation in routine work is limited by high costs and long processing times. In the interim, microarray technology offers an attractive alternative, demonstrated amply in animal $(14,20,29,31,57)$ and 
plant systems $(10-12,25,43,45,53,58,60)$. The only significant drawback of microarray detection systems are the specialized technological resources (equipment) required and the costs of fabricating chips. Macroarray methods require less in the way of specialized equipment and may be a technically more amenable alternative $(3,21,35,36,39,46,51)$.

We recently reported the development of a robust macroarray platform and methods for the detection of five grapevine-infecting members of the family Closteroviridae that established a proofof-principal for the unbiased multiplex detection of most viruses associated with grapevine leafroll disease (51). The objective of this work has been to develop a more comprehensive, extended array with probes for as many as 38 viruses, including the most significant grapevine-infecting viruses with regard to both prevalence and emergence. In a screen of 99 symptomatic and asymptomatic grapevines, from Europe and the United States, the method detected representative virus species of the four main families in 46 plants, of which $35 \%$ were shown to be mixed infections, thereby demonstrating the overall utility of this cropspecific macroarray. This technology is appropriate for routine diagnostics of high value planting stocks, in particular for foundation plant and certification programs, and in quarantine centers to facilitate the international exchange of germplasm.

\section{MATERIALS AND METHODS}

Plants, RNA extraction, and serological testing. From 2010 to 2012, cane or leaf tissue was collected from 99 symptomatic and asymptomatic cultivated grapevine selections growing in 10 locations in the United States and Europe. Total RNA was extracted from this material using the CTAB method of Gambino et al. (24) with downstream modifications as previously described (51). ELISA was carried out using antibodies to the following viruses listed in Table 1: Grapevine leafroll-associated virus 1 (GLRaV-1), Grapevine leafroll-associated virus 2 (GLRaV-2), Grapevine leafroll-associated virus 3 (GLRaV-3), Grapevine leafroll-associated virus 4 (GLRaV-4) strain 5, GLRaV-4 strain 6, Grapevine leafroll-associated virus 7 (GLRaV-7), generic antibodies to GLRaV-4/9 (=GLRaV-4, GLRaV-4 strain 5, GLRaV-4 strain 6, GLRaV-4 strain 9 and GLRaV-7), Grapevine virus A (GVA), Grapevine fleck virus (GFkV), Strawberry latent ringspot virus (SLRSV), Grapevine fanleaf virus (GFLV), Arabis mosaic virus (ArMV), Tomato ringspot virus (ToRSV), Tobacco ringspot virus (TRSV), Raspberry ringspot virus (RpRSV), and Tomato blackring virus (TBRV). All antibodies were obtained from Bioreba (Reinach, Switzerland) except those against GLRaV-4 strain 5 (from AC Diagnostics, Fayetteville, AR) and used according to the manufacturer's instructions.

Reverse transcription, cDNA amplification, and enzyme labeling. Four hundred nanograms of total plant RNA for each sample was reverse-transcribed using $200 \mathrm{U}$ of M-MLV reverse transcriptase (Invitrogen, Carlsbad, CA) with $1 \mu \mathrm{M}$ random anchored primer (5'-TGGTAGCTCTTGATCANNNNN-3') (3) and $1 \mathrm{mM}$ dNTPs in a $20 \mu \mathrm{l}$ volume, according to the manufacturer's instructions. Five nanograms of an internal control transcript prepared from the cloned complementary DNA fragment of West Nile virus (WNV) (51) was spiked into each reaction. Once the reverse transcription reaction was complete, $0.5 \mu \mathrm{l}$ was used as the template for PCR amplification using IQ supermix (BioRad, Hercules, CA) with $0.2 \mu \mathrm{M}$ of the above random anchored primer in combination with $0.8 \mu \mathrm{M}$ anchor primer $\left(5^{\prime}\right.$-AGAGTTGG TAGCTCTTGATC-3'). Thermocycler conditions were as follows: $94^{\circ} \mathrm{C}$ for $5 \mathrm{~min} ; 94^{\circ} \mathrm{C}$ for $30 \mathrm{~s}, 55^{\circ} \mathrm{C}$ for $1 \mathrm{~min}, 72^{\circ} \mathrm{C}$ for $2 \mathrm{~min}$ ( $\times 40) ; 72^{\circ} \mathrm{C}$ for $7 \mathrm{~min}$, after which $400 \mathrm{ng}$ of the purified amplified cDNA, quantified spectrophotometrically, was denatured by boiling in a total volume of $20 \mu \mathrm{l}$ of sterile distilled water for 5 min and placed immediately on ice. The denatured cDNA was labeled with alkaline phosphatase by adding the components of the AlkPhos Direct Labeling and Detection System with CDPStar (GE Healthcare Bio-Sciences Corp., Pittsburgh, PA): $20 \mu \mathrm{l}$ of reaction buffer, $16 \mu \mathrm{l}$ of sterile distilled water, $4 \mu \mathrm{l}$ of labeling reagent, $4 \mu \mathrm{l}$ of crosslinker, and incubating for $30 \mathrm{~min}$ at $37^{\circ} \mathrm{C}$.

Oligonucleotide probes. Probes for all viruses were designed both in the plus and minus viral sense and were derived from three sources (Table 1, Supplemental Table 1): (i) in-house 70mers (ii) 70-mer oligonucleotides designed for a microarray (19), and (iii) 60-mer oligonucleotides designed for a microarray (10). In-house probes were designed by visual selection (based on the most conserved regions with the most sequence information) from consensus sequences obtained by aligning all available NCBI database sequences for each virus species as described previously (51). Control probes were ribosomal RNA specific rRNA-1, -5, -6 , ST-1 trimer, and WNV-2, details of which are described elsewhere (51), along with plant-specific probes (52). Most oligonucleotides were spotted at a final concentration of $20 \mu \mathrm{M}$, while the rRNA probes were at $2 \mu \mathrm{M}$. The printing procedure was carried out as described previously (2). Two arrays are described in this report, the first designated Grapearray 2 and a revised and updated version designated Grapearray4. The general organization of the probes on the nylon membrane for both the arrays is shown in Figure 1 with exact details of probe positions shown in Supplemental Figure 1.

Hybridization and visualization. Membranes were stripped by incubating at $60^{\circ} \mathrm{C}$ in $0.5 \%$ (wt/vol) sodium dodecyl sulfate (SDS) for 1 to $2 \mathrm{~h}$ followed by washing in $0.1 \mathrm{M}$ Tris- $\mathrm{HCl}, \mathrm{pH}$ 8.0 , for $5 \mathrm{~min}$. Prehybridization for $1 \mathrm{~h}$ was done in $10 \mathrm{ml}$ of the hybridization buffer supplied with the Alkphos Direct kit (GE Healthcare, Piscataway, $\mathrm{NJ}$ ) at $55^{\circ} \mathrm{C}$, after which all the labeled PCR product from the AlkPhos labeling reaction $(64 \mu \mathrm{l})$ and an internal control of $0.5 \mathrm{ng}$ of AlkPhos labeled ST-1 trimer (51) were added to $5 \mathrm{ml}$ of hybridization buffer and applied to the membrane. Hybridization was done at $55^{\circ} \mathrm{C}$ overnight. Membranes were washed twice in a primary wash buffer $(2 \mathrm{M}$ urea, $0.1 \% \mathrm{SDS}, 50 \mathrm{mM}$ sodium phosphate, $150 \mathrm{mM} \mathrm{NaCl}$, and $0.2 \%$ [wt/vol] blocking agent supplied with the Alkphos Direct kit) at $55^{\circ} \mathrm{C}$ for $15 \mathrm{~min}$ each, followed by washing in a secondary wash buffer (50 mM Tris, $100 \mathrm{mM} \mathrm{NaCl}, \mathrm{pH} \mathrm{10.0)}$ for $10 \mathrm{~min}$, at room temperature on a shaker. The membranes were incubated with CDP-star chemiluminescent (GE Healthcare) reagent for $5 \mathrm{~min}$, drained, and viewed both in a Biospectrum 500 Imager (UVP, Upland, CA) for 10 to $45 \mathrm{~min}$ and by exposing to Chemiluminescence Bio Max film (Kodak Co., Rochester, NY) overnight. Samples were only considered positive for a virus if more than one oligonucleotide spotted pair gave a signal. Membranes were stored moist at $4{ }^{\circ} \mathrm{C}$ sealed in a plastic bag and reused 20 to 30 times.

PCR detection of viruses using conserved generic and species-specific primers. For confirmation of viruses not detected by ELISA, previously described generic and speciesspecific primers were employed, namely Tymoviridae (TMZ-F: 5'-GGSCCMGTSAARAARTAYCA-3' and TMZ-R: 5'-GCCA GRTTGTARTCRGRGTTG-3') (50), GLRaV-2 (L2F: 5'-ATAATT CGGCGTACATCCCCACTT-3' and U2R: 5'-GCCCTCCGCG CAACTAATGACAG-3') (8), GLRaV-3 (LC1-F: 5'-CGCTAGG GCTGTGGAAGTATT-3' and LC2-R: 5'-GTTGTCCCGGGTAC CAGATAT-3') (37), GLRaV-4 strains (LRAmp-F: 5'-ATTTAGG TAATGTWGTRGCTAC-3' and LRAmp-R: 5'-TATCCTCAGW GAGGAARCGG-3') (1), GLRaV-7 (LR7-F: 5'-TATATCCCA ACGGAGATGGC-3' and LR7-R 5'-ATGTTCCTCCACCAAAAT CG-3') (19) GVA (GVA-F: 5'-GACAAATGGCACACTACG-3' and GVA-R: 5'-AGCCTGACCTAGTCATCTT-3'), GVB (GVB-F: 5'-GTGCTAAGAACGTCTTCACAGC-3' and GVB-R: 5'-ATC AGCAAACACGCTTGAACCG-3') (42) and GVE (GVE-1-For: 5'-AATGGAGTCAAAAGCGATCC-3' and GVE-Rev: 5'-GTAG GGTCAATCAACCAACA-3' (16). RT-PCR was done with primer pairs specific to each virus using the same conditions described in 
the publication that initially reported them, using random hexamers (Invitrogen) to generate cDNA. RT and PCR were done with M-MLV reverse transcriptase (Invitrogen, Carlsbad, CA) and iQ Supermix (BioRad), respectively.

\section{RESULTS AND DISCUSSION}

ELISA and macroarray testing of grapevines. A total of 99 vines were received as dormant canes or sampled as in season

TABLE 1 . List of the virus taxa represented on the array and the corresponding number of probes per taxon organized by designer

\begin{tabular}{|c|c|c|c|c|c|c|c|}
\hline Family $^{\mathrm{a}}$ & Genus & Taxa and groups ${ }^{b}$ & Acronym & Cornell & Engel et al. & UPVM & Total \\
\hline \multirow[t]{10}{*}{ Betaflexiviridae } & $\ldots$ & Betaflexiviridae & & 0 & 0 & 10 & 10 \\
\hline & Foveavirus & Foveavirus & & 0 & 0 & 10 & 10 \\
\hline & & Grapevine rupestris stem pitting-associated virus & GRSPaV & 8 & 9 & 4 & 21 \\
\hline & Trichovirus & Trichovirus & & 0 & 0 & 10 & 10 \\
\hline & & Grapevine pinot gris virus* & GPGV & 11 & 0 & 0 & 11 \\
\hline & & Grapevine berry inner necrosis virus & GINV & 10 & 0 & 2 & 12 \\
\hline & Vitivirus & Grapevine virus $A$ & GVA & 10 & 5 & 2 & 17 \\
\hline & & Grapevine virus B & GVB & 10 & 5 & 2 & 17 \\
\hline & & Grapevine virus $D$ & GVD & 10 & 0 & 2 & 12 \\
\hline & & Grapevine virus $E$ & GVE & 10 & 0 & 2 & 12 \\
\hline \multirow[t]{2}{*}{ Bromoviridae } & Alfamovirus & Alfalfa mosaic virus & AMV & 10 & 0 & 0 & 10 \\
\hline & Cucumovirus & Cucumber mosaic virus & CMV & 12 & 0 & 0 & 12 \\
\hline Bunyaviridae & Tospovirus & Tomato spotted wilt virus & TSWV & 15 & 0 & 0 & 15 \\
\hline Caulimoviridae & Badnavirus & Grapevine vein clearing virus & GVCV & 11 & 0 & 0 & 11 \\
\hline \multirow[t]{12}{*}{ Closteroviridae } & $\ldots$ & Ampelo/Clostero & & 6 & 0 & 31 & 37 \\
\hline & Ampelovirus & Grapevine leafroll associated virus -1 & GLRaV-1 & 8 & 8 & 2 & 18 \\
\hline & & Grapevine leafroll associated virus-3 & GLRaV-3 & 9 & 11 & 2 & 22 \\
\hline & & Grapevine leafroll associated virus-4 strain $\mathrm{Pr}$ & GLRaV-4 strain Pr & 6 & 0 & 3 & 9 \\
\hline & & Grapevine leafroll associated virus-4 strain 9 & GLRaV-4 strain 9 & 16 & 5 & 2 & 23 \\
\hline & & Grapevine leafroll associated virus -4 & GLRaV-4 strain 4 & 16 & 6 & 2 & 24 \\
\hline & & Grapevine leafroll associated virus-4 strain 5 & GLRaV-4 strain 5 & 16 & 0 & 2 & 18 \\
\hline & & Grapevine leafroll associated virus-4 strain 6 & GLRaV-4 strain 6 & 13 & 0 & 2 & 15 \\
\hline & & Grapevine leafroll associated virus-4 strain De & GLRaV-4 strain De & 4 & 0 & 0 & 4 \\
\hline & & Grapevine leafroll associated virus-4 strain Carnelian* & $\begin{array}{l}\text { GLRaV-4 strain } \\
\text { Carnelian }\end{array}$ & 8 & 0 & 0 & 8 \\
\hline & Velarivirus & Grapevine leafroll associated virus-7 & GLRaV-7 & 19 & 3 & 2 & 24 \\
\hline & Closterovirus & Grapevine leafroll associated virus-2 & GLRaV-2 & 11 & 7 & 2 & 20 \\
\hline Geminiviridae & $\ldots$ & Grapevine red blotch associated virus*c & GRBaV & 10 & 0 & 0 & 10 \\
\hline Luteoviridae & Enamovirus & Grapevine enamovirus* & & 7 & 0 & 0 & 7 \\
\hline Reoviridae & Oryzavirus & Grapevine oryzavirus* & & 8 & 0 & 0 & 8 \\
\hline \multirow[t]{18}{*}{ Secoviridae } & $\ldots$ & Comovirinae & & 0 & 0 & 10 & 10 \\
\hline & $\ldots$ & Secoviridae & & 0 & 0 & 10 & 10 \\
\hline & Nepovirus & Nepovirus Subgroup A & & 0 & 0 & 10 & 10 \\
\hline & & Nepovirus Subgroup B & & 0 & 0 & 10 & 10 \\
\hline & & Nepovirus Subgroup C & & 0 & 0 & 10 & 10 \\
\hline & & Nepovirus & & 0 & 0 & 10 & 10 \\
\hline & & Arabis mosaic virus & ArMV & 9 & 7 & 0 & 16 \\
\hline & & Grapevine Anatolian ringspot virus & GARSV & 10 & 0 & 4 & 14 \\
\hline & & Grapevine Bulgarian latent virus & GBLV & 11 & 0 & 0 & 11 \\
\hline & & Grapevine chrome mosaic virus & GCMV & 10 & 0 & 2 & 12 \\
\hline & & Grapevine deformation virus & GDefV & 10 & 0 & 4 & 14 \\
\hline & & Grapevine fanleaf virus & GFLV & 8 & 7 & 4 & 19 \\
\hline & & Peach rosette mosaic virus & PRMV & 10 & 0 & 2 & 12 \\
\hline & & Raspberry ringspot virus & RpRSV & 10 & 0 & 6 & 16 \\
\hline & & Strawberry latent ringspot virus & SLRSV & 10 & 0 & 6 & 16 \\
\hline & & Tobacco ringspot virus & TRSV & 10 & 0 & 4 & 14 \\
\hline & & Tomato blackring virus & TBRV & 10 & 0 & 5 & 15 \\
\hline & & Tomato ringspot virus & ToRSV & 10 & 0 & 6 & 16 \\
\hline \multirow[t]{3}{*}{ Tombusviridae } & $\ldots$ & Tombusviridae & & 0 & 0 & 10 & 10 \\
\hline & Tombusvirus & Tombusvirus & & 0 & 0 & 10 & 10 \\
\hline & & Grapevine Algerian latent virus & GALV & 10 & 0 & 2 & 12 \\
\hline \multirow[t]{8}{*}{ Tymoviridae } & $\ldots$ & Tymoviridae & & 0 & 0 & 8 & 8 \\
\hline & Maculavirus & Grapevine fleck virus & GFkV & 10 & 0 & 2 & 12 \\
\hline & & Grapevine redglobe virus & GRGV & 10 & 0 & 2 & 12 \\
\hline & Marafivirus & Marafivirus & & 0 & 0 & 10 & 10 \\
\hline & & Grapevine Syrah virus-1* & GSyV-1 & 12 & 0 & 0 & 12 \\
\hline & & Grapevine asteroid mosaic-associated virus & GAMaV & 10 & 0 & 2 & 12 \\
\hline & & Grapevine rupestris vein feathering virus & GRVFV & 10 & 0 & 2 & 12 \\
\hline & Tymovirus & Tymovirus & & 0 & 0 & 5 & 5 \\
\hline \multirow[t]{3}{*}{ Unassigned } & Ideaovirus & Raspberry bushy dwarf virus & RBDV & 9 & 0 & 3 & 12 \\
\hline & & TOTAL VIRUS SPECIES & TOTAL PROBES & 463 & 73 & 253 & 789 \\
\hline & & & & & \multicolumn{2}{|c|}{ PLUS+MINUS } & 1,578 \\
\hline
\end{tabular}

\footnotetext{
a The first four columns indicate the taxa or acronym/designation. The latter four columns indicate the number of probes organized by the source of the design; Cornell, those probes designed as part of this study and those (GLRaV-specific) of Thompson et al. (51); Engel et al. probes described by Engel et al. (19); and UPVM, probes designed as part of the Universal Plant Virus Microarray (Bagewadi et al., 2010) (10). See Supplemental Table 1 for details.

b Those taxa marked with an asterisk * were entirely new to Grapearray4. All other taxa acronyms/designations were present on both Grapearray 2 and Grapearray 4.

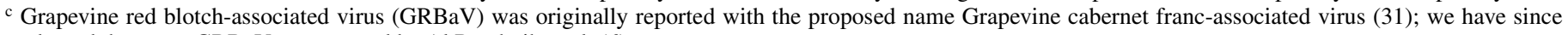
adopted the name GRBaV as proposed by Al Rwahnih et al. (6).
} 


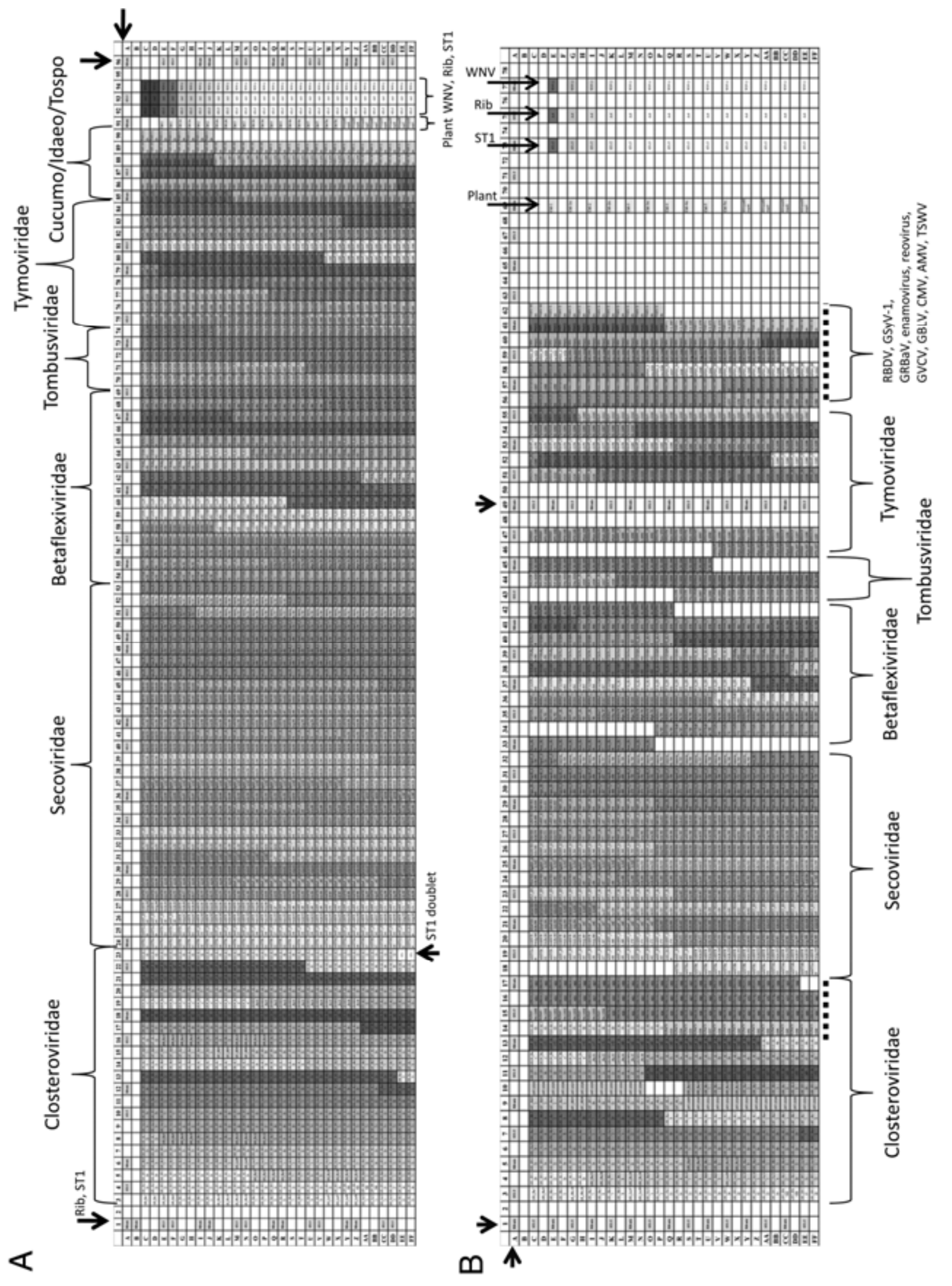

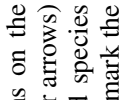

突记

으을

20. 을

政

放

言完

웜

콘

o

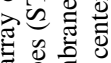

을 을

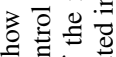

列

可

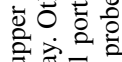

政

๘

自

要

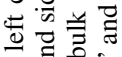

包

을 을

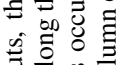

詝

형

홍 응을 :

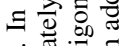

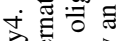

을

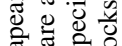

의의

ㅅ․

흘

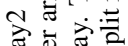

क्ष

政

5ै

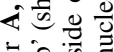

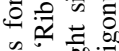

on

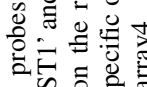

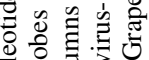

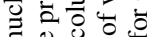

总: 田品

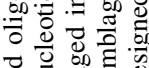

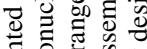

然些

낭

ㄹㅇㅝ

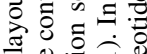

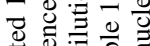

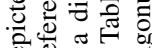

원. $\cong$ ज

局可

궁

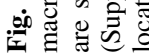


growth. While many were selected based on aberrant growth, some were presumed uninfected (e.g., imported materials from foreign centers) and no systematic and multi-season assessment of symptoms was attempted. Each sample was tested by ELISA for the presence of 16 viruses, most of which are of importance in certification programs. The samples were also tested using two macroarray platforms (designated Grapearray2 and Grapearray4) for the multiplex detection of 32 or more recently 38 viruses of grapevine. The ELISA and macroarray results are summarized in Table 2. The viruses indicated as being detected in the macroarray are those for which hybridization was observed to two or more oligonucleotide probes with sequences of that virus. The detection of the virus in the array was confirmed for at least one of the viruses in many but not all of the samples by either ELISA or by

TABLE 2. Grapevine samples testing positive for virus in the array with the detected viruses indicated

\begin{tabular}{|c|c|c|c|c|}
\hline Sample ${ }^{a}$ & Vitis species cultivar ${ }^{\mathrm{b}}$ & Collection site $(\text { origin })^{c}$ & ELISA $^{\mathrm{d}}$ & Grapearray \\
\hline CF17P2 & Vitis vinifera 'Cabernet Franc' & New York, USA & Negative & (GRGV, GRVFV) \\
\hline CFP6V2 & Vitis vinifera 'Cabernet Franc' & New York, USA & Negative & (GRSPaV, GRVFV) \\
\hline $17-30$ & Vitis vinifera 'Riesling' & New York, USA & Negative & Tymoviridae \\
\hline $17-44 \mathrm{~L}$ & Vitis vinifera 'Riesling' & New York, USA & Negative & (GRSPaV), Tymoviridae \\
\hline $17-44 R$ & Vitis vinifera 'Riesling' & New York, USA & Negative & Tymoviridae \\
\hline $20-56$ & Vitis vinifera 'Riesling' & New York, USA & Negative & GRVFV, GRGV, Tymoviridae \\
\hline $22-21^{a}$ & Vitis vinifera 'Riesling' & New York, USA & Negative & $\begin{array}{l}\text { (GAMaV, GRGV, GRVFV, } \\
\text { GSyV-1), Tymoviridae }\end{array}$ \\
\hline GLRaV-4 GRdL ${ }^{a}$ & Vitis vinifera 'Thompson Seedless' & New York, USA (Switzerland) & GLRaV-4/9 & (GAMaV, GSyV-1), Tymoviridae \\
\hline LR2 Y206 & Vitis vinifera 'Chaouch rose' & New York, USA (France) & GLRaV-2 & GLRaV-2, (GSyV-1) \\
\hline NY $124,5 B B+$ & Vitis vinifera 'Gerwurtraminer' & New York, USA (France) & GFLV & GFLV, Tymoviridae \\
\hline NY121, 5BB & Vitis vinifera 'Chardonnay' & New York, USA (France) & Negative & Tymoviridae \\
\hline NY122, 5BB+ & Vitis vinifera 'Chardonnay' & New York, USA (France) & GFLV & GFLV, Tymoviridae \\
\hline TJB5-9 & Vitis vinifera 'Cabernet Franc' & New York, USA & Negative & GRSPaV, Tymoviridae \\
\hline LR3 Y285 & Vitis vinifera 'Raziki Y285' & New York, USA (France) & GLRaV-3 & GLRaV-3 \\
\hline C-14 & Vitis vinifera 'Cabernet Franc' & New York, USA & Negative & Tymoviridae \\
\hline LN33 & Vitis sp. & New York, USA & Negative & $(\mathrm{GAMaV})$ \\
\hline GLRaV-7 VSV & Vitis sp. & California, USA & Negative & $\begin{array}{r}\text { (GLRaV-4, GLRaV-6, GLRaV-9, } \\
\text { GLRaV-Pr, GRSPaV, GRVFV) }\end{array}$ \\
\hline LR6 Estellat & Vitis sp. & California, USA & GLRaV-3 & GLRaV-3, (GRSPaV) \\
\hline PN23a & Vitis sp. & California, USA & Negative & GLRaV-7, (GRSPaV) \\
\hline LR6 SCH5 & Vitis sp. & California, USA & GLRaV-5, GLRaV-6 & $\begin{array}{l}\text { GLRaV-5, (GRSPaV), (GVA), } \\
\text { Tymoviridae }\end{array}$ \\
\hline $125 \mathrm{~V} 1$ & Vitis sp. 'Millardet et de Grasset 125-1' & New York, USA & Negative & $(\mathrm{GRSPaV})$ \\
\hline $188-15 \# 3$ & Vitis sp. 'Castel 188-15' & New York, USA & Negative & GRGV, Tymoviridae \\
\hline $3309 \# 30$ & Vitis sp. 'Couderc 3309' & New York, USA & Negative & (GAMaV, GRVFV) \\
\hline $3309-V 16^{\mathrm{a}}$ & Vitis sp. 'Couderc 3309' & New York, USA & Negative & GAMaV, GSyV-1 \\
\hline $01-63^{\mathrm{a}}$ & Vitis sp. 'Galibert 133-6' & New York, USA (France) & TRSV & (GRSPaV), TRSV \\
\hline $02-54$ & Vitis sp. 'Veeport' & New York, USA & Negative & GVE \\
\hline $05-47$ & Vitis sp. 'Perbos 155 ' & New York, USA (France) & ToRSV & ToRSV \\
\hline $05-92^{\mathrm{a}}$ & Vitis sp. 'Bertille-Seyve 3408' & New York, USA (France) & ToRSV & ToRSV \\
\hline $06-72$ & Vitis sp. 'Bertille-Seyve 2862' & New York, USA (France) & TRSV & TRSV \\
\hline $07-46$ & Vitis riparia & New York, USA & Negative & $(\mathrm{GAMaV})$ \\
\hline $08-53$ & Vitis sp. 'Noah' & New York, USA (Florida) & ArMV & ArMV \\
\hline $09-95$ & Vitis sp. 'Goethe' & New York, USA (Massachusetts) & Negative & (GFLV) \\
\hline $1-82$ & Vitis sp. 'Seibel 6339' & New York, USA (France) & Negative & $(\mathrm{GVB}, \mathrm{GVE})$ \\
\hline $10-51^{\mathrm{a}}$ & Vitis rupestris 'Alphonse de Serres' & New York, USA (Texas) & GFLV & (ArMV), GFLV \\
\hline $12-77$ & Vitis sp. 'Seibel 14.654' & New York, USA (France) & Negative & $(\mathrm{GRSPaV})$ \\
\hline $12-97$ & Vitis sp. 'Ill 559-1' & New York, USA (Illinois) & GLRaV-1, GLRaV-3 & GLRaV-3 \\
\hline $14-21$ & Vitis sp. 'Geisenheim 26' & New York, USA (Germany) & ArMV & ArMV, (ToRSV) \\
\hline $14-32^{\mathrm{a}}$ & Vitis sp. 'Bailey Alicante A' & New York, USA (Japan) & Negative & GLRaV-3 \\
\hline $14-55$ & Vitis vinifera 'Thompson No. 5' & New York, USA & Negative & GLRaV-3, (GRVFV) \\
\hline $17-5$ & Vitis sp. 'Agria' & New York, USA & Negative & $(\mathrm{GAMaV})$ \\
\hline $17-9$ & Vitis sp. 'Landot 6222' & New York, USA & GFLV & GFLV \\
\hline ArMV Syrah & Vitis vinifera 'Syrah' & New York, USA (France) & ArMV & ArMV \\
\hline HAA\#1 & Vitis labrusca 'Concord' & New York, USA & GLRaV-3 & GLRaV-3 \\
\hline НАА\#8 & Vitis labrusca 'Concord' & New York, USA & GLRaV-3 & GLRaV-3, GVE \\
\hline DAA\#2 & Vitis labrusca 'Concord' & New York, USA & GLRaV-3 & GLRaV-3, GVA, GVB, GVE \\
\hline DAA\#3 & Vitis labrusca 'Concord' & New York, USA & GLRaV-3 & GLRaV-3, GVB, GVE \\
\hline
\end{tabular}

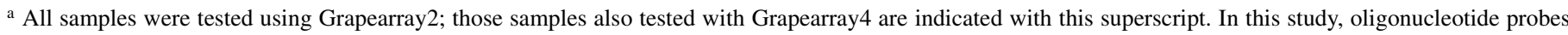
specific to GSyV-1 were the only probes new to Grapearray4 that tested positive. In all other instances, those viruses detected in Grapearray 2 were also detected in Grapearray4.

b Cultivar refers to the cultivar or clone name, where known.

c Origin refers to the most recent state or country where the clone was obtained; the original source, where known, is indicated in parentheses.

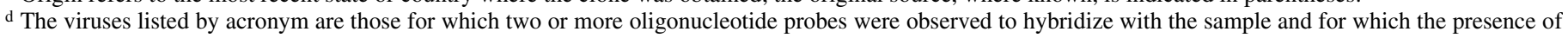
the virus was confirmed by enzyme-linked immunosorbent assay (ELISA) or polymerase chain reaction (PCR). GLRaV-4, GLRaV-5, GLRaV-6, GLRaV-9, and GLRaV-Pr refer to GLRaV-4 strain 4, GLRaV-4 strain 5, GLRaV-4 strain 6, GLRaV-4 strain 9, and GLRaV-4 strain Pr, respectively. GLRaV-4/9 indicates virus detected by a mixture of generic antibodies to GLRaV-4 strains and GLRaV-7. Parentheses indicate array results for which efforts were not made to confirm the presence of the virus or viruses by PCR or ELISA. Two or more viruses together in parentheses indicates the possibility of cross hybridization in the array due to sequence identity. Acronyms and viruses are as follow: ArMV - Arabis mosaic virus; GAMaV - Grapevine asteroid mosaic virus; GFkV - Grapevine fleck virus; GFLV - Grapevine fanleaf virus; GLRaV-1, -2, -3, -4, and -7 - Grapevine leafroll associated virus-1, -2, -3, -4, and -7; GRGV - Grapevine redglobe virus; GRSPaV - Grapevine rupestris stem pitting associated virus; GRVFV - Grapevine rupestris vein feathering virus; GSyV-1 - Grapevine syrah virus-1; GVA,-B, and -E - Grapevine virus A, B, E; ToRSV - Tomato ringspot virus, TRSV - Tobacco ringspot virus. Note that signals from generic family probes are not included unless a corresponding virus species was not identified. Samples (GLRaV-4 GRdL, 12-97, and LR6 SCH5) testing positive for viruses in ELISA but not in the array were also tested by RT-PCR. The former two samples' disease status was confirmed; while LR6 SCH5 was negative. 
RT-PCR amplification and sequencing. (In cases where the indicated viruses were not confirmed by an independent method, the acronyms in Table 2 are in parentheses.) For 18 (or 24) of the viruses in Grapearray2 (or Grapearray4), ELISA detection reagents were not available. Confirmations by RT-PCR were made using generic primers designed to amplify members within a genus or family when available (e.g., Nepovirus or Tymoviridae), although the subsequent sequencing of the products identified the virus to species. In no case were the array results not able to be confirmed by RT-PCR. Vines were sampled over a 3-year period and in different seasons; therefore, it is not entirely unexpected that a nepovirus or leafroll-associated virus might be detected by ELISA, but not in the array, or vice versa. By contrast, samples processed at the same time and tested by both ELISA and in the array yielded consistent results.

Grapearray 2 contained probes for the detection of 32 viruses (Fig. 1A, Table 1) and this array was employed in the initial testing of all vines. Using sequence information that became available in 2011 and 2012, additional probes were designed resulting in the printing of Grapearray4, with probes specific to 38 virus species (Fig. 1B). Grapearray4 was used to retest a subset of nine samples (Table 2). Both arrays have generic and family level probes designed to hybridize with multiple species and to function in detecting conserved sequences that may be present in previously uncharacterized viruses in the same genera and families. Thus, in some cases such as for members of the family Tymoviridae, only family level probes hybridized and the identity of the putative virus was not established.

With the current methodologies, the macroarrays are thought to provide a sensitivity comparable to ELISA (3), although this will vary depending on the specific antibody reagents employed. Viruses detected in ELISA were largely detected in the arrays and vice versa (Table 2 ) to the extent that antibodies were available. In cases where a virus was indicated by the array, but not seen by ELISA, RT-PCR was employed to confirm the presence of the virus (or noted otherwise in Table 2).

Virus population profiles. Of the 99 samples collected and tested, 46 were found positive for virus infection using the array (Table 2). Of those, 30 were positive for a single virus and the remaining 16 for two or more viruses (Fig. 2A). The types of viruses detected represented members of each of the four main virus families present in grapevines with most to least being species of the family Tymoviridae (23 samples), Betaflexiviridae (15 samples), Closteroviridae (13 samples), and Secoviridae (13

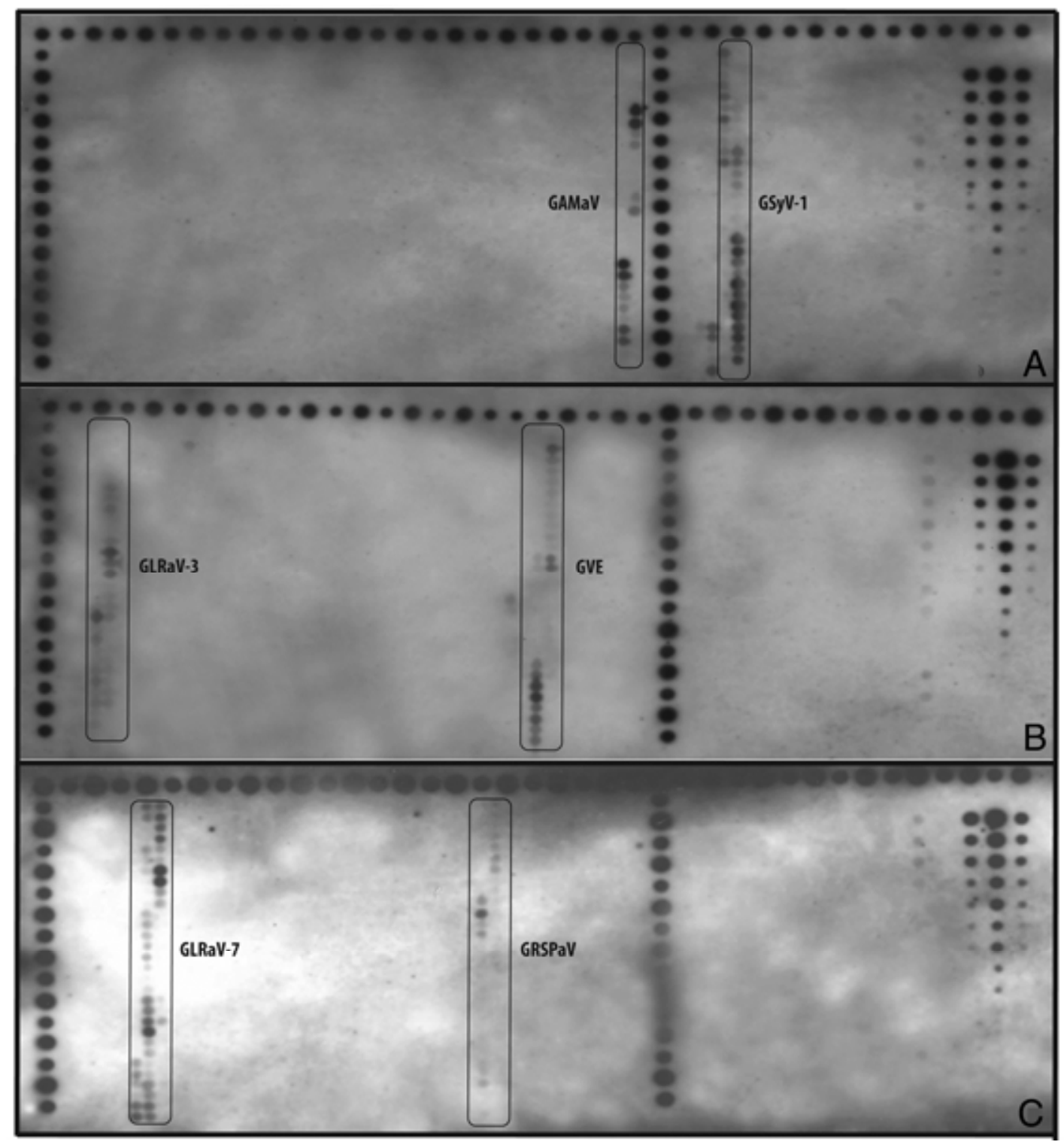

Fig. 2. Representative examples of Grapearray4 results for three virus-infected grapevine samples; A, 3306-V16, B, HAA\#8 Concord, and C, PN23. GAMaV Grapevine asteroid mosaic-associated virus. GSyV-1 - Grapevine syrah virus 1, GLRaV-3 - Grapevine leafroll-associated virus 3, GVE - Grapevine virus E, GLRaV-7 - Grapevine leafroll-associated virus 7, and GRSPaV - Grapevine rupestris stem pitting-associated virus. 
samples). Every possible paired combination of each family was found, except closterovirus/secovirus. Almost half of the betaflexivirids found, particularly vitiviruses, were in combination with members of the family Closteroviridae, presumably reflecting their shared mode of transmission via mealybug vectors (17, 33). By contrast, tymovirids and secovirids are generally vectored by beetles and nematodes, respectively, although for all grapevine-infecting tymovirids no vector has been described (40). The most commonly found virus was GLRaV-3 from the genus Ampelovirus in the family Closteroviridae, which was detected in nine plants, consistent with previous reports on the prevalence of GLRaV-3 in grapevine in North America $(23,38,41)$. These results are presumably biased by the nonrandom nature of the sampling that was frequently based on symptoms that may or may not have been due to the viruses detected.

The most commonly detected viruses were members of the family Tymoviridae, viruses that represent the fleck complex $(40,49)$. Of the four main grapevine-infecting genera, the impact and distribution of the fleck complex is the least understood; infection is assumed to be latent or semi-latent with some indication of adverse effects $(27,40)$. The results of the macroarray suggest that tymovirids are potentially more common in grape- vine than previously thought. Of the 23 positive samples, nine were in mixed infections with viruses from other genera, while at least another two (20-56 and 3309-V16) (Table 2) comprised mixed infections of distinct tymovirus species. Despite the paucity of data available on the effects of infection, the possible implications of a mixed infection involving tymoviruses was recently demonstrated for Syrah decline where a combination of two tymoviruses, GSyV-1 and GRVFV, and a foveavirus, GRSPaV, were found (5). The array detection of the tymoviruses GAMaV and GRGV in New York vines was confirmed by the sequencing of PCR products in samples 3309-V16, 20-56, and 188-15\#3. Globally to date, GAMaV was thought to be limited to California, while GRGV has only been reported for Italian and Albanian vines (47).

Array performance. The array technology used in this study can be described as a continual work in progress, with the constant discovery of new viruses requiring the design and addition of oligonucleotide probes, extending the printed area on the membrane. The formats described here are an adaptation of a previous design establishing a proof-of-principal as reported by Thompson et al (51). The main changes in design have been governed by a limitation in the actual size of the array. In all the

\section{A}

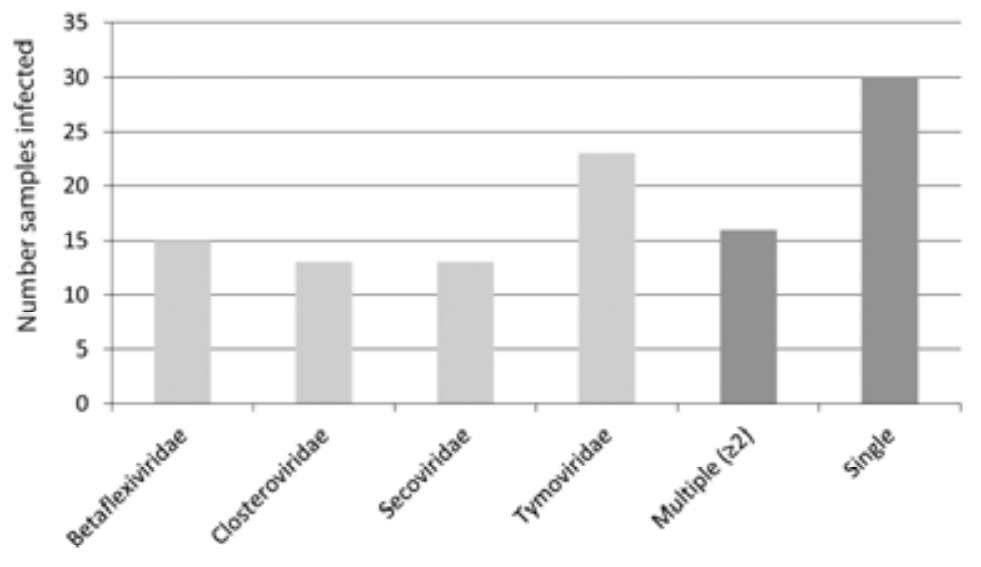

B

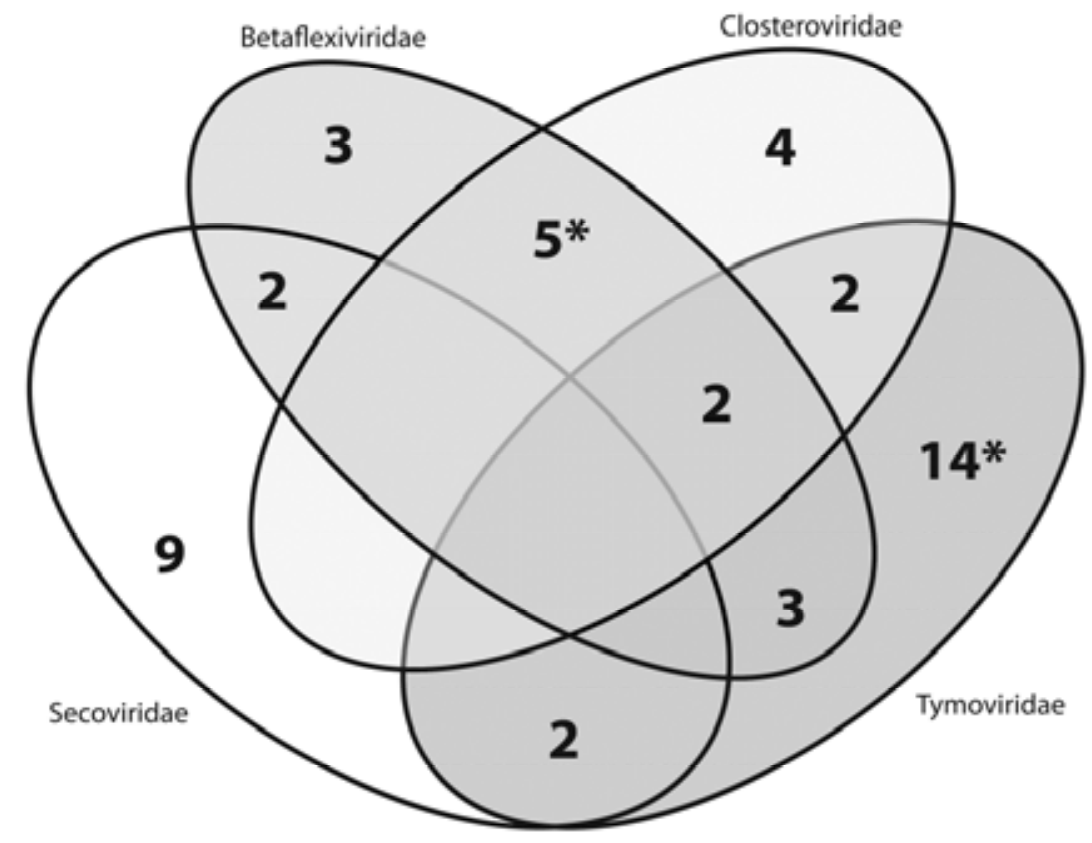

Fig. 3. Virus prevalence illustrated as samples infected by members of different virus families and as single and multiple ( $\geq 2)$ viruses. A, Histogram of the number of grapevine samples positive for each member of the four main grapevine-infecting virus families, and as single and multiple $(\geq 2)$ infections. B, Venn diagram showing the distribution of virus infections by family, alone or in combinations for all grapevine samples testing positive. * indicates a mixed infection of two viruses from the same family. 
initial macroarrays developed in our lab, including Grapearray2, oligonucleotides have been spotted in pairs to minimize ambiguity over signal interpretation $(2,3,51)$. In order to conserve space and recognizing the utility of using both sense and antisense oligonucleotides, the layout of oligonucleotides in Grapearray4 involves a shift to spotting single sense and antisense oligonucleotides as pairs, rather than duplicating the spotting of each individual probe. As the design evolves, it is likely to undergo further space-reducing measures such as a refined selection of optimal oligonucleotides for detection based on empirical results and the plethora of software available for microarray applications that consider a range of characteristics including $\mathrm{G}+\mathrm{C}$ content, melting temperature $(\mathrm{Tm})$, secondary structure, position in transcript, sequence complexity, probe-target identity and the length of identity stretches $(26,34,44,48)$. In the design of oligonucleotides for the present arrays, we were often limited by sequence availability, although our principle criterion in selection was based on having as broad a detection capacity as possible within virus species and families so as to afford detection of novel but related viruses; the priority has been to avoid false negatives, not to identify viruses to species. We concentrated on selecting oligonucleotides in the most sequenced regions of each viral genome where identity among isolates was greater than $85 \%$ (26).

A further consideration in development is the use of plant specific oligonucleotides that provide a useful visual gauge for the relative sensitivity in each array experiment (Fig. 3). Without these, assessment of the integrity of the original plant derived RNA has been made using the ribosomal specific oligonucleotides (Rib mix in Figures 1 and 2 and Supplemental Figures 1 and 2 ), which although confirming the correct processing of plant derived RNA, are in such abundance that minor RNAs can be overlooked. The inclusion of these plant-specific oligonucleotides is even more pertinent given the fact that rRNA subtraction methods aimed at increasing the sensitivity of the array are under development. Improving sensitivity of the array, which is at present comparable with ELISA (4), is particularly desirable for diagnosing low titer and/or DNA viruses. Tests using the present array on samples positive for the newly discovered geminiviridaelike $\operatorname{GRBaV}(6,32)$ have largely proven negative, most likely because of lower viral transcripts present in infected tissue, being solely transcript-derived rather than genomic and subgenomically derived as in the case for RNA viruses. Problems in the detection of putatively low-titer viruses may simply be overcome by greater sequence information as illustrated here for Grapevine leafroll associated virus-7 (GLRaV-7) from the proposed genus Velarivirus in the family Closteroviridae. In the previous proof-ofprincipal format (51), sequence information for GLRaV-7 was limited to around 600 bases. Since then the full sequence of GLRaV-7 $(7,28)$ with around 16,500 nucleotides has allowed the design of new GLRaV-7 specific oligonucleotides to be incorporated into the present array providing a greater breadth of detection (Fig. 2). Of the 38 viruses for which probes were designed and spotted on the array, 16 were detected in this survey with all the main virus families being well-represented. These numbers alone illustrate the potential of the array. The only alternative detection methods to provide this level of information would be large-scale sequencing with bioinformatic analyses; while powerful, these methods are not yet in common use. By employing the macroarray approach for both experimental and commercial field samples we demonstrate its practicable utility in not only providing useful information on grapevine virus populations but also as a standard diagnostic tool in phytosanitary and certification schemes. Adoption of the array in quarantine centers would offer the potential to expedite the release of material introduced from foreign sources. It would be especially useful to identify cultivar introductions in need of virus elimination therapy; a decision to process material for virus curing could be shortened from the 2 to 3 years necessary for grafting bioassays to a few days. Indeed, as part of this study, the macroarray has been useful in confirming the virus negative status of grapevine introductions into North America, of two Lemberger clones from Austria and one Riesling clone from Germany.

\section{ACKNOWLEDGMENTS}

We thank P. Das, N. Kamath, R. Cox, and D. MacUmber for their support in the lab. We are indebted to M. Al Rwahnih and A. Rowhani of Foundation Plant Services, University of California at Davis, O. Lemaire, INRA, Colmar, France, V. Colova-Tsolova, Florida A\&M University, and C. Balmelli, Agroscope, Changins, Switzerland for providing some of the samples tested and to Sead Sabanadzovic, Mississippi State University, for providing unpublished data. This work was supported in part by funding from the U.S. Department of Agriculture Animal Plant Health Inspection Service as part of the National Clean Plant Network program and by Agriculture and Food Research Initiative Competitive Grants nos. 2009-55605-05184 and 2009-55605-05023 from the USDA National Institute of Food and Agriculture.

\section{LITERATURE CITED}

1. Abou Ghanem-Sabanadzovic, N., Sabanadzovic, S., Gugerli, P., and Rowhani, A. 2012. Genome organization, serology and phylogeny of Grapevine leafroll-associated viruses 4 and 6: Taxonomic implications. Virus Res. 163:120-128.

2. Agindotan, B., and Perry, K. L. 2007. Macroarray detection of plant RNA viruses using randomly primed and amplified complementary DNAs from infected plants. Phytopathology 97:119-127.

3. Agindotan, B., and Perry, K. L. 2008. Macroarray detection of eleven potato-infecting viruses and Potato spindle tuber viroid. Plant Dis. 92: 730-740.

4. Agindotan, B. O., Shiel, P. J., and Berger, P. H. 2007. Simultaneous detection of potato viruses, PLRV, PVA, PVX and PVY from dormant potato tubers by TaqMan real-time RT-PCR. J. Virol. Methods 142:1-9.

5. Al Rwahnih, M., Daubert, S., Golino, D., and Rowhani, A. 2009. Deep sequencing analysis of RNAs from a grapevine showing Syrah decline symptoms reveals a multiple virus infection that includes a novel virus. Virology 387:395-401.

6. Al Rwahnih, M., Dave, A., Anderson, M. M., Rowhani, A., Uyemoto, J. K., and Sudarshana, M. 2013. Association of a DNA virus with grapevines affected by red blotch disease in California. Phytopathology 103:1069-1076.

7. Al Rwahnih, M., Dolja, V. V., Daubert, S., Koonin, E. V., and Rowhani, A. 2012. Genomic and biological analysis of Grapevine leafroll-associated virus 7 reveals a possible new genus within the family Closteroviridae. Virus Res. 163:302-329.

8. Alkowni, R., Rowhani, A., Daubert, S., and Golino, D. 2004. Partial characterization of a new ampelovirus associated with grapevine leafroll disease. J. Plant Pathol. 86:123-133.

9. Arnold, K., Golino, D. A., and McRoberts, N. 2012. Statistical parameters of spatial patterns of spread for leafroll disease. Pages 192-193 in: 17th Congress of the ICVG. B. Ferguson, ed. Foundation Plant Services, University of California, Davis, CA.

10. Bagewadi, B., Fischer, K., Henderson, D. C., Jordan, R. L., Wang, D., Perry, K. L., Melcher, U., Hammond, J., and Fauquet, C. M. 2010. Universal plant virus microarray development and validation. (Abstr.) Phytopathology 100(suppl.):S154

11. Boonham, N., Walsh, K., Smith, P., Madagan, K., Graham, I., and Barker, I. 2003. Detection of potato viruses using microarray technology: Towards a generic method for plant viral disease diagnosis. J. Virol. Methods 108:181-187.

12. Bystricka, D., Lenz, O., Mraz, I., Piherova, L., Kmoch, S., and Sip, M. 2005. Oligonucleotide-based microarray: A new improvement in microarray detection of plant viruses. J. Virol. Methods 128:176-182.

13. Charles, J. G., Froud, K. J., van den Brink, R., and Allan, D. J. 2009. Mealybugs and the spread of grapevine leafroll-associated virus 3 (GLRaV-3) in a New Zealand vineyard. Austral. Plant Pathol. 38:576-583.

14. Chizhikov, V., Wagner, M., Ivshina, A., Hoshino, Y., Kapikian, A. Z., and Chumakov, K. 2002. Detection and genotyping of human group A rotaviruses by oligonucleotide microarray hybridization. J. Clin. Microbiol. 40:2398-2407.

15. Cid, M., Pereiro, S., Cabaleiro, C., and Segura, A. 2010. Citrus mealybug (Hemiptera: Pseudococcidae) movement and population dynamics in an arbor-trained vineyard. J. Econ. Entomol. 103:619-630.

16. Coetzee, B., Maree, H. J., Stephan, D., Freeborough, M. J., and Burger, J. T. 2010. The first complete nucleotide sequence of a grapevine virus $\mathrm{E}$ 
variant. Arch. Virol. 155:1357-1360.

17. Credi, R., and Giunchedi, L. 1996. Grapevine leafroll-associated viruses and grapevine virus A in selected Vitis vinifera cultivars in northern Italy. Plant Pathol. 45:1110-1116.

18. Demangeat, G., Komar, V., Van-Ghelder, C., Voisin, R., Lemaire, O., Esmenjaud, D., and Fuchs, M. 2010. Transmission competency of singlefemale Xiphinema index lines for Grapevine fanleaf virus. Phytopathology 100:384-389.

19. Engel, E. A., Escobar, P. F., Rojas, L. A., Rivera, P. A., Fiore, N., and Valenzuela, P. D. 2010. A diagnostic oligonucleotide microarray for simultaneous detection of grapevine viruses. J. Virol. Methods 163:445-451.

20. Erlandsson, L., Rosenstierne, M. W., McLoughlin, K., Jaing, C., and Fomsgaard, A. 2011. The microbial detection array combined with random Phi29-amplification used as a diagnostic tool for virus detection in clinical samples. PLoS One 6:e22631.

21. Fessehaie, A., De Boer, S. H., and Levesque, C. A. 2003. An oligonucleotide array for the identification and differentiation of bacteria pathogenic on potato. Phytopathology 93:262-269.

22. Fuchs, M., Marsella-Herrick, P., Loeb, G. M., Martinson, T. E., and Hoch, H. C. 2009. Diversity of ampeloviruses in mealybug and soft ccale vectors and in grapevine hosts from leafroll-affected vineyards. Phytopathology 99:1177-1184.

23. Fuchs, M., Martinson, T. E., Loeb, G. M., and Hoch, H. C. 2009. Survey for the three major leafroll disease-associated viruses in finger lakes vineyards in New York. Plant Dis. 93:395-401.

24. Gambino, G., Perrone, I., and Gribaudo, I. 2008. A rapid and effective method for RNA extraction from different tissues of grapevine and other woody plants. Phytochem. Anal. 19:520-525.

25. Grover, V., Pierce, M. L., Hoyt, P., Zhang, F., and Melcher, U. 2010. Oligonucleotide-based microarray for detection of plant viruses employing sequence-independent amplification of targets. J. Virol. Methods 163:57-67.

26. He, Z. L., Wu, L. Y., Li, X. Y., Fields, M. W., and Zhou, J. Z. 2005. Empirical establishment of oligonucleotide probe design criteria. Appl. Environ. Microbiol. 71:3753-3760.

27. Hewitt, W. B., Goheen, A. C., Raski, D. J., and Gooding, G. V. 1962. Studies on virus diseases of the grapevine in California. Vitis 3:57-83.

28. Jelkmann, W., Mikona, C., Turturo, C., Navarro, B., Rott, M. E., Menzel, W., Saldarelli, P., Minafra, A., and Martelli, G. P. 2012. Molecular characterization and taxonomy of grapevine leafroll-associated virus 7 . Arch. Virol. 157:359-362.

29. Klaassen, C. H., Prinsen, C. F., de Valk, H. A., Horrevorts, A. M., Jeunink, M. A., and Thunnissen, F. B. 2004. DNA microarray format for detection and subtyping of human papillomavirus. J. Clin. Microbiol. 42:21522160 .

30. Komar, V., Vigne, E., Demangeat, G., and Fuchs, M. 2007. Beneficial effect of selective virus elimination on the performance of Vitis vinifera cv. Chardonnay. Am. J. Enol. Vitic. 58:202-210.

31. Kozal, M. J., Shah, N., Shen, N., Yang, R., Fucini, R., Merigan, T. C., Richman, D. D., Morris, D., Hubbell, E., Chee, M., and Gingeras, T. R. 1996. Extensive polymorphisms observed in HIV-1 clade B protease gene using high-density oligonucleotide arrays. Nat. Med. 2:753-759.

32. Krenz, B., Thompson, J. R., Fuchs, M., and Perry, K. L. 2012. Complete genome sequence of a new circular DNA virus from grapevine. J. Virol. 86:7715.

33. Le Maguet, J., Beuve, M., Herrbach, E., and Lemaire, O. 2012. Transmission of six ampeloviruses and two vitiviruses to grapevine by Phenacoccus aceris. Phytopathology 102:717-723.

34. Lemoine, S., Combes, F., and Le Crom, S. 2009. An evaluation of custom microarray applications: The oligonucleotide design challenge. Nucleic Acids Res. 37:1726-1739.

35. Levesque, C. A., Harlton, C. E., and de Cock, A. W. 1998. Identification of some oomycetes by reverse dot blot hybridization. Phytopathology 88:213-222.

36. Lievens, B., Brouwer, M., Vanachter, A. C., Levesque, C. A., Cammue, B. P., and Thomma, B. P. 2003. Design and development of a DNA array for rapid detection and identification of multiple tomato vascular wilt pathogens. FEMS Microbiol. Lett. 223:113-122.

37. Ling, K. S., Zhu, H. Y., Drong, R. F., Slightom, J. L., McFerson, J. R., and Gonsalves, D. 1998. Nucleotide sequence of the 3'-terminal two-thirds of the grapevine leafroll-associated virus-3 genome reveals a typical monopartite closterovirus. J. Gen. Virol. 79:1299-1307.
38. MacKenzie, D. J., Johnson, R. C., and Warner, C. 1996. Incidence of four important viral pathogens in Canadian vineyards. Plant Dis. 80:955-958.

39. Maoka, T., Sugiyama, S., Maruta, Y., and Hataya, T. 2010. Application of cDNA macroarray for simultaneous detection of 12 potato viruses. Plant Dis. $94: 1248-1254$.

40. Martelli, G. P., and Boudon-Padieu, E. 2006. Directory of infectious diseases of grapevines. In: Options Mediterraneennes. G. P. Martelli and E. Boudon-Padieu, eds. CIHEAM, Bari.

41. Martin, R. R., Eastwell, K. C., Wagner, A., Lamprecht, S., and Tzanetakis, I. E. 2005. Survey for viruses of grapevine in Oregon and Washington. Plant Dis. 89:763-766.

42. Minafra, A., and Hadidi, A. 1994. Sensitive detection of grapevine virusA, virus-B, or leafroll-associated-3 from viruliferous mealybugs and infected tissue by cDNA amplification. J. Virol. Methods 47:175-187.

43. Nicolaisen, M. 2011. An oligonucleotide-based microarray for detection of plant RNA viruses. J. Virol. Methods 173:137-143.

44. Nielsen, H. B., Wernersson, R., and Knudsen, S. 2003. Design of oligonucleotides for microarrays and perspectives for design of multitranscriptome arrays. Nucleic Acids Res. 31:3491-3496.

45. Pasquini, G., Barba, M., Hadidi, A., Faggioli, F., Negri, R., Sobol, I., Tiberini, A., Caglayan, K., Mazyad, H., Anfoka, G., Ghanim, M., Zeidan, M., and Czosnek, H. 2008. Oligonucleotide microarray-based detection and genotyping of Plum pox virus. J. Virol. Methods 147:118-126.

46. Perry, K. L., and Lu, X. 2010. A tospovirus new to North America: Virus detection and discovery through the use of a macroarray for viruses of solanaceous crops. (Abstr.) Phytopathology 100(suppl.):S100.

47. Plant Virology in Sub-Saharan Africa. 2001. in: Plant Virology in SubSaharan Africa. J. d'A. Hughes, and B. O. Odu, eds. International Institute of Tropical Agriculture Ibadan, Nigeria.

48. Rouillard, J. M., Zuker, M., and Gulari, E. 2003. OligoArray 2.0: Design of oligonucleotide probes for DNA microarrays using a thermodynamic approach. Nucleic Acids Res. 31:3057-3062.

49. Sabanadzovic, S., Abou-Ghanem, N., Castellano, M. A., Digiaro, M., and Martelli, G. P. 2000. Grapevine fleck virus-like viruses in Vitis. Arch. Virol. 145:553-565.

50. Sabanadzovic, S., Abou Ghanem-Sabanadzovic, N., and Gorbalenya, A. E. 2009. Permutation of the active site of putative RNA-dependent RNA polymerase in a newly identified species of plant alpha-like virus. Virology 394:1-7.

51. Thompson, J. R., Fuchs, M., Fischer, K. F., and Perry, K. L. 2012. Macroarray detection of grapevine leafroll-associated viruses. J. Virol. Methods 183:161-169.

52. Thompson, J. R., Wetzel, S., Klerks, M. M., Vaskova, D., Schoen, C. D., Spak, J., and Jelkmann, W. 2003. Multiplex RT-PCR detection of four aphid-borne strawberry viruses in Fragaria spp. in combination with a plant mRNA specific internal control. J. Virol. Methods 111:85-93.

53. Tiberini, A., Tomassoli, L., Barba, M., and Hadidi, A. 2010. Oligonucleotide microarray-based detection and identification of 10 major tomato viruses. J. Virol. Methods 168:133-140.

54. Tsai, C. W., Chau, J., Fernandez, L., Bosco, D., Daane, K. M., and Almeida, R. P. 2008. Transmission of grapevine leafroll-associated virus 3 by the vine mealybug (Planococcus ficus). Phytopathology 98:1093-1098.

55. Tsai, C. W., Rowhani, A., Golino, D. A., Daane, K. M., and Almeida, R. P. 2010. Mealybug transmission of grapevine leafroll viruses: An analysis of virus-vector specificity. Phytopathology 100:830-834.

56. Villate, L., Fievet, V., Hanse, B., Delemarre, F., Plantard, O., Esmenjaud, D., and van Helden, M. 2008. Spatial distribution of the dagger nematode Xiphinema index and its associated Grapevine fanleaf virus in French vineyard. Phytopathology 98:942-948.

57. Wang, D., Coscoy, L., Zylberberg, M., Avila, P. C., Boushey, H. A., Ganem, D., and DeRisi, J. L. 2002. Microarray-based detection and genotyping of viral pathogens. Proc. Natl. Acad. Sci. USA 99:15687-15692.

58. Wei, T., Pearson, M. N., Blohm, D., Nolte, M., and Armstrong, K. 2009. Development of a short oligonucleotide microarray for the detection and identification of multiple potyviruses. J. Virol. Methods 162:109-118.

59. Zhang, Y., Singh, K., Kaur, R., and Qiu, W. 2011. Association of a novel DNA virus with the grapevine vein-clearing and vine decline syndrome. Phytopathology 101:1081-1090.

60. Zhang, Y., Yin, J., Li, G., Li, M., Huang, X., Chen, H., Zhao, W., and Zhu, S. 2010. Oligonucleotide microarray with a minimal number of probes for the detection and identification of thirteen genera of plant viruses. J. Virol. Methods 167:53-60. 